\title{
Orientierungen und Praktiken sportunterrichtlicher Differenzkonstruktionen. Perspektiven praxistheoretischer Unterrichtsforschung im formal inklusiven Grundschulsport
}

\author{
Daniel Schiller $\cdot$ Daniel Rode $\cdot$ Benjamin Zander $\cdot$ Dennis Wolff
}

Eingegangen: 1. Juni 2020 / Angenommen: 12. Oktober 2020 / Online publiziert: 12. Januar 2021

(C) Der/die Autor(en) 2021

Zusammenfassung Im Beitrag werden Differenzkonstruktionen und praktische Ein-/Ausschlussprozesse im formal inklusiven Grundschulsportunterricht untersucht. Auf Basis videographischer Daten wird in einer wissenssoziologischen, praxistheoretischen Perspektive an einem Fallbeispiel ein kollektiv-geteilter Orientierungsrahmen der Personifikation unterrichtlicher Handlungen aufgezeigt, an dem drei Dimensionen von Differenzkonstruktionen - Reifizierung von ,Unfähigkeit‘, Komplementarität von Handlungsräumen, Verschränkung von Sachund Differenzherstellung - sowie Perspektiven für eine praxistheoretische Grundschul(sport)unterrichtsforschung diskutiert werden können.

Schlüsselwörter Sportunterricht · Inklusion · Praxistheorie · Differenzforschung · Dokumentarische Methode $\cdot$ Konjunktiver Erfahrungsraum

Dr. phil. D. Schiller $(\bowtie)$

Institut für Sport- und Bewegungswissenschaften, Universität Osnabrück,

Jahnstr. 75, 49080 Osnabrïck, Deutschland

E-Mail: daniel.schiller@uni-osnabrueck.de

Ass.-Prof. Dr. phil. D. Rode

Interfakultärer Fachbereich Sport- und Bewegungswissenschaft/USI, Universität Salzburg,

Schlossallee 49, 5400 Hallein/Rif, Salzburg, Österreich

E-Mail: daniel.rode@sbg.ac.at

Dr. phil. B. Zander

Institut für Sportwissenschaften, Georg-August-Universität Göttingen,

Sprangerweg 2, 37075 Göttingen, Deutschland

E-Mail: benjamin.zander@uni-goettingen.de

Dr. phil. D. Wolff

Institut für Sportwissenschaft, Stiftung Universität Hildesheim,

Universitätsplatz 1, 31141 Hildesheim, Deutschland

E-Mail: wolffd@uni-hildesheim.de 


\title{
Orientations and practices of doing difference in PE lessons. Perspectives of praxeological research in primary school inclusive PE classes
}

\begin{abstract}
The paper investigates practices of doing difference and ensuing processes of practical exclusion in primary school inclusive PE classes. Based on videographic data, the praxeological analysis of a case lesson reveals a collective orientation framework of 'personification of actions'. We draw on this example to discuss three key dimensions of the practical construction of differences-reification of 'disability', complementary possibilities of action, relations between doing content and doing difference-, while mapping out perspectives of praxeological (physical education) primary education research.
\end{abstract}

Keywords Physical education - Inclusion · Practice theory · Doing difference · Documentary method · Conjunctive space of experience

\section{Einleitung}

Inklusion hat sich in der Sportpädagogik vom bildungspolitischen Auftrag zum vieldiskutierten pädagogischen und fachwissenschaftlichen Thema entwickelt. Eine leitende Maxime für die schulische Umsetzung ist im deutschsprachigen wie internationalen Raum die gemeinsame Beschulung von Schüler*innen mit und ohne ,Behinderung ' im ,Regelsportunterricht' (Block et al. 2017, S. 233 f.). Im sportpädagogischen Diskurs finden sich hierfür didaktische, anwendungsbezogene Beiträge (z.B. Giese und Weigelt 2015a,b, 2017; Ruin et al. 2016) ${ }^{1}$, empirische Untersuchungen zu individuellen Perspektiven der beteiligten Akteur*innen (Block et al. 2017) sowie programmatische, grundlagentheoretische und kritisch-hermeneutische Beiträge (z. B. Giese 2019; Haegele et al. 2020; Erhorn et al. 2020). Bildungspolitische Aufträge und pädagogische Ansprüche der Inklusion stellen die konkrete Praxis des Sportunterrichts vor besondere Herausforderungen, die auch an ihre fachliche Spezifik geknüpft sind, etwa die herausgehobene Bedeutung von Körperlichkeit und körperlich zur Aufführung gebrachten Verhaltensweisen und Fähigkeiten (vgl. Block et al. 2017, S. 236) oder die gesellschaftlichen Zuschreibungen und etablierten Praxen bezogen auf die Sache ,Sport“ (vgl. Giese 2019, S. 9; $133 \mathrm{ff}$.). Vor allem zeigt sich aber, dass diese fachliche Praxis des formal inklusiven Sportunterrichts - das Wie der Inklusionsbemühungen von Lehrkräften und Schüler*innen - noch weitgehend unerforscht ist.

Der Beitrag setzt an dieser Leerstelle an. Auf Basis videographischen Datenmaterials wird rekonstruiert, wie in der Praxis formal inklusiven Grundschulportunterrichts Inklusionsbemühungen konkret ausgestaltet werden und wie mit diesen Bemühungen wiederrum bestimmte Differenzsetzungen und praktisch wirksame Ein-/ Ausschlussmechanismen einhergehen. Zugrunde liegt eine praxistheoretische Analyseperspektive, die zuerst eingeführt und bezogen auf den sportpädagogischen und

\footnotetext{
1 Vgl. auch die Themenhefte Sportunterricht 6/2012, Sportpädagogik 6/2013, Sportpraxis 9+10/2013.
} 
erziehungswissenschaftlichen Forschungsdiskurs verortet wird (2). Im Anschluss an die Praxeologische Wissenssoziologie (Bohnsack 2017) besteht die Grundausrichtung darin, sich nicht mit individuellen Sichtweisen der Akteur*innen auf den formal inklusiven Unterricht (z.B. Ruin und Meier 2018) zu begnügen, sondern die kollektiv getragenen Praktiken, Orientierungen und Strukturen des Unterrichts zu rekonstruieren, in die diese individuellen Perspektiven eingelassen sind. Nach der Darstellung der Methodik einer dokumentarischen Videoanalyse (3) verdichten und diskutieren wir im Hauptteil unsere Untersuchungsergebnisse in Hinblick auf Dimensionen sportunterrichtlicher Differenzkonstruktionen (4). Dies erfolgt an einem Fallbeispiel im Horizont des geteilten Orientierungsrahmens - der „Personifikation unterrichtlicher Handlungen“ -, der fallspezifisch rekonstruiert wurde und die fallspezifische Differenzordnung kennzeichnet. Im Fazit werden Perspektiven des praxistheoretischen Ansatzes für inklusionsbezogene Sportunterrichtsforschung aufgezeigt (5).

\section{Inklusion als soziale Praxis - Theoretische Perspektive und Verortung im Forschungsdiskurs}

\subsection{Inklusion - Differenzkonstruktion - Ein-/Ausschlussprozesse}

In der Sportpädagogik wird die Inklusionsdebatte stark anwendungsorientiert geführt (siehe Fn. 1), zunehmend entstehen aber Anschlüsse an internationale Diskurse zur Adapted Physical Activity und Adapted Physical Education sowie an behindertenund inklusionspädagogische Diskurse (Block et al. 2017). In der inklusionspädagogischen Forschung setzt sich - im Spektrum zwischen ,weiten', eine grundlegende Heterogenität entlang intersektionaler Kategorien (z.B. ,Geschlecht') betonenden Perspektiven einerseits und ,engen', auf ,Behinderung ' bzw. ,sonderpädagogischen Förderbedarf‘ fokussierenden Verständnissen andererseits (vgl. zu dieser Einteilung Budde et al. 2016) - eine sozialkonstruktivistische Perspektive durch (vgl. Sturm und Wagner-Willi 2015, S. 66), an die wir anschließen: ,Behinderung ' wird nicht absolut gesetzt, sondern als Resultat wiederholter sozialer Zuschreibungen von Un-/ Fähigkeiten (Dis-/Ability) und damit verbundenen Prozessen des Ein- und Ausschlusses verstanden, die in Relation zu ebenso sozial konstruierten Normen und Normalitäten stehen (z. B. Buchner et al. 2015; Herzmann und Merl 2019). Inklusion ist damit nicht ohne Exklusion zu denken (Budde und Hummrich 2013) und kann in der Schule mit Blick auf Dynamiken und Formen von bildungsrelevanten Ein- und Ausschlüssen betrachtet werden, die sich auf das soziale Miteinander wie auf das fachliche Lerngeschehen beziehen können (Wagner-Willi und Sturm 2012). ${ }^{2}$ Vor der Annahme, dass Ein-/Ausschlussprozesse insbesondere ,über die Konstruktion und Bearbeitung von Differenzen in sozialen Interaktionen und Praktiken“ (Sturm und

\footnotetext{
2 Die Forschungsperspektive muss so auch nicht Schüler*innen mit zugeschriebenem ,Förderbedarf" integrierenden Unterricht fokussieren, sondern eignet sich generell für heterogenitäts- und ungleichheitssensible Unterrichtsforschung.
} 
Wagner-Willi 2015, S. 66) entstehen, setzten die empirischen Rekonstruktionen an diesen Differenzherstellungen auf der Interaktionsebene des Unterrichts an.

Fokussiert man in dieser Perspektive die Kategorie ,Förderbedarf“ bzw. ,Dis-/ Ability“, zeigt sich, dass diese in vielen Fällen auch praktisch zur zentralen Differenzkategorie avanciert, entlang derer in den unterrichtlichen Handlungen der Beteiligten wertende, Beteiligungsmöglichkeiten moderierende Unterscheidungen und Klassifizierungen hergestellt werden (Budde et al. 2016). In anderen Klassen spielt diese Kategorie dagegen keine Rolle für faktisch vollzogene Differenzsetzungen; diese orientieren sich vielmehr an differenten, unterrichtsbezogenen Kategorien wie ,Leistung ' oder ,Verhalten“ und Prinzipien wie ,Arbeitsgeschwindigkeit' (ebd.). Fokussiert man diese Kategorien, wird deutlich, wie sie im einen Fall zur Verstärkung und Verfestigung der Leitdifferenz ,behindert/nicht behindert' führen (z. B. werden ,förderbedürftige‘ Schüler*innen bei Regelverstößen anders adressiert als ,Regelschüler*innen'; vgl. auch Merl 2019). In anderen Fällen avancieren Verhaltensoder Leistungsdifferenzen (z. B. in der Dimension gut/schlecht) selbst zur Leitunterscheidung, entlang derer Schüler*innen positioniert werden. Im Einzelfall kommt es hier zu komplexen Überlagerungen, die auch Kategorien wie ,Geschlecht ‘ und ,Peerkultur' einschließen (vgl. z. B. Sturm und Wagner-Willi 2015).

Diese Befunde beziehen sich auf den ,Klassenraumunterricht' unterschiedlicher Schulstufen (auch der Grundschule) und sind nicht ohne weiteres auf den Sportunterricht und seine fachliche Spezifik (siehe Einleitung) übertragbar - entsprechende sportpädagogische Studien stehen bislang noch aus. Der beschriebenen sozialkonstruktivistischen Perspektive wird nur in Einzelstudien gefolgt, die in einem ableismus-kritischen Zugang (hierzu Lindmeier und Buchner 2019) Exklusionspotenziale insbesondere im Hinblick auf normative Körper- und Subjektideale auf den Ebenen fachdidaktischer Konzepte, bildungspolitischer Programme und den Einstellungen von Lehrkräften herausarbeiten (Giese und Ruin 2018). Gerade letztgenannte Analysen sind einer individuumszentrierten Forschungsrichtung zuzuordnen, die insgesamt einen Großteil des nationalen wie internationalen sportpädagogischen Forschungsstandes ausmacht: Interviewstudien zu den individuellen Sichtweisen von Eltern, Lehrpersonen, Schüler*innen ohne und - noch sehr wenig - auch mit ,Behinderung' liefern wichtige Einsichten in die subjektiven Erfahrungen und Deutungen der Akteur*innen zum formal inklusiven Sportunterricht (im Überblick Block et al. 2017, S. 235 ff.).

Komplementierend zur dominanten sportpädagogischen Forschungsausrichtung und anknüpfend an die skizzierten Befunde einer praxistheoretischen Inklusionsforschung blicken wir auf praktisch vollzogene Inklusionsbemühungen im Grundschulsportunterricht - auf das beobachtbare Tun, so wie es sich im Unterricht zwischen den Akteur*innen körperlich, sprachlich und durch materielle Dinge entfaltet (Schatzki 2002) - und rekonstruieren Dimensionen hierbei erfolgender Differenzkonstruktionen.

\subsection{Interaktiver Erfahrungsraum - Handlungsprobleme - Orientierungen}

Zur analytischen Konzeptionalisierung unterrichtlicher Praxis knüpfen wir an die Praxeologische Wissenssoziologie (Mannheim 1964; Bohnsack 2017) an, die sich 
gegenwärtig in der Beforschung formal inklusiver Schulsettings theoretisch (z.B. Sturm 2016) und empirisch (z. B. Sturm und Wagner-Willi 2015; Sturm et al. 2020) ausdifferenziert. Sie betont, dass die Unterrichtspraxis - und damit die von uns fokussierten Differenzkonstruktionen - maßgeblich über atheoretisches Wissen, implizite Gesetzmäßigkeiten und vorreflexive Orientierungen organisiert ist. Leitend ist ein Verständnis von Sportunterricht als interaktiven Erfahrungsraum, der durch konkrete unterrichtliche Vollzüge hergestellt wird (Bohnsack 2017, S. $121 \mathrm{ff}$; Zander 2018) und durch spezifische Handlungsprobleme und Erlebnisstrukturen gekennzeichnet ist, die von den Akteur*innen sowohl fraglos vorausgesetzt als auch gemäß präreflexiver Muster stets neu erzeugt und modifiziert werden.

Die praktische Bearbeitung sportunterrichtlicher Handlungsprobleme erfolgt nicht willkürlich oder rein individuell, sondern ist von mehr oder weniger geteilten Rahmen und Wissensordnungen orientiert, die im Vollzug aufgerufen und situativ aktualisiert werden. Die Rekonstruktion dieser Rahmungen und Orientierungen folgt der wissenssoziologischen Leitunterscheidung (vgl. hierzu Mannheim 1964) von immanenten Sinngehalten einerseits, die sich entlang theoretischer, kommunikativer Wissensbestände der Akteur*innen konstituieren, z. B. Themen von Interaktionen, didaktischer Programmatik, Regeln des Unterrichts oder Normerwartungen der Organisation. Andererseits konturiert sich der geteilte Erfahrungsraum des Sportunterrichts durch einen Dokumentsinn, der aus dem praktischen Vollzug selbst emergiert und seinerseits die Art und Weise orientiert, wie immanente Sinn- und Bedeutungsgehalte hervorgebracht und verhandelt, d.h. wie Themen bearbeitet, Programmatik umgesetzt, Rollen eingenommen, Regeln (nicht) eingehalten und Erwartungen (nicht) erfüllt werden.

In dieser wissenssoziologischen Ausdeutung einer sozialkonstruktivistischen, praxisanalytischen Inklusionsperspektive lässt sich formal inklusiver Sportunterricht - durch den sportpädagogischen Forschungsstand bedingt noch in weitem Fokus daraufhin befragen, wie Differenzen praktisch hergestellt und bearbeitet werden, auf welche Weise damit Ein- und Ausschlüsse einhergehen und inwiefern dadurch ein interaktiver Erfahrungsraum sowie spezifische Handlungsprobleme kollektiv hervorgebracht und bearbeitet werden.

\section{Methodik}

Methodisch unmittelbar anschlussfähig an die wissenssoziologisch fundierte Analyseperspektive gehen die nachfolgenden exemplarisch verdichteten Ergebnisse auf Videoanalysen nach der Dokumentarischen Methode (Bohnsack 2010; Asbrand und Martens 2018) in einem größeren Projektzusammenhang zurück. ${ }^{3}$ Auf einer imma-

\footnotetext{
3 Hintergrund ist das von den Verfassern geleitete, eigenfinanzierte Forschungsprojekt ,Sportpädagogische Perspektiven praxistheoretischer Unterrichtsforschung“, das mit Sekundäranalysen videographischer Daten praxistheoretische Forschungsperspektiven für Sportunterrichtsforschung fruchtbar machen möchte. Der Gesamtkorpus umfasst Sportunterrichtsaufnahmen unterschiedlicher Klassenstufen und Schultypen aus früheren Projekten der Verfasser, wissenschaftlichen Videoportalen (z. B. HILDE) und weiteren Forschungsprojekten. Die Daten zum Fallbeispiel wurden freundlicherweise vom BMBF-geförderten Projekt „Qualifizierung von Sportlehrkräften für einen inklusiven Sportunterricht“ unter Leitung von Prof. Dr. Jan Erhorn bereitgestellt.
} 
nenten Sinnebene wurde nach theoretischen Wissensbeständen der Differenzerzeugung gefragt, die sich in den Unterrichtsinteraktionen kommunikativ ausdrücken und interpretieren lassen, z. B. der thematische Sinngehalt einer Regel oder einer Norm. Darüber hinaus richtet sich auf einer dokumentarischen Sinnebene der Blick auf atheoretisches Wissen, das intuitiv die Handlungspraxis koordiniert und damit beispielsweise implizite Umgangsweisen der Regelauslegung rahmt sowie - aufgrund des konjunktiven Charakters dieser Wissensdimension - ein unmittelbares Verstehen unterrichtlicher Ordnungen ermöglicht.

Das Videomaterial wurde zunächst in Interaktionseinheiten phasiert, anschließend in Bezug auf die verbale Kommunikation wörtlich transkribiert und in Hinblick auf die sprachliche, räumliche, körperliche und materielle Verfasstheit sozialer Situationen in genauen Situationsbeschreibungen und Stillstellungen aufbereitet (Asbrand und Martens 2018). Einzelne Sequenzen wurden dann einer formulierenden Feininterpretation und einer sinngenetisch reflektierenden Rekonstruktion unterzogen (siehe hierzu auch Bohnsack 2010). Dabei wurden neben der Anfangspassage gerade jene Handlungseinheiten ausgewählt, die eine hohe interaktive Dichte aufweisen, d.h. eine Eingebundenheit mehrerer Akteure*innen mit zum Teil besonderem Engagement. In diesen Situationen kommen verstärkt Relevanzsysteme der Beteiligten zur Aufführung (dazu Asbrand und Martens 2018, S. 53f.). Die reflektierende Interpretation erfolgte als Sequenzanalyse (vgl. ebd., S. 104ff.), in der die interaktive Organisation des unterrichtlichen Handelns in einem Dreischritt von Proposition, Elaboration und Konklusion mit Blick auf zugrundeliegende Handlungsmodi (univok, komplementär, oppositionell etc.; vgl. ebd.) untersucht wurde.

\section{Dimensionen von Differenzkonstruktionen am Beispiel der Personifikation unterrichtlicher Handlungen}

Am Fallbeispiel sollen nun wichtige Dimensionen von Differenzkonstruktionen im kollektiven, interaktiv hergestellten Erfahrungsraum des Grundschulsportunterrichts aufgezeigt und diskutiert werden. Die Beispielsstunde stammt aus dem Sportunterricht einer vierten Klasse, in dem ,Regelschüler*innen“ und zwei Schüler*innen (Lea und Tobi) mit formal zugeschriebenem ,Förderbedarf' gemeinsam von einer Lehrperson (L) ohne Hinzunahme weiterer Fachkräfte unterrichtet werden. ${ }^{4}$ Das auf kollektive Begeisterung stoßende Stundenthema ist ,ne Runde Brennball“ (L). Nachdem gemeinsam die Regeln erinnert und Sonderregeln für Lea und Tobi eingeführt werden, teilt L zwei Teams ein und geht mit Lea die Handlungsschritte des Brennballspiels noch einmal individuell durch, indem beide gemeinsam den Parkour durchlaufen. Es folgt eine gut fünfzehnminütige Spielphase, in der beide Teams zwischendurch die Rollen (Wurf- und Fangteam) wechseln. Lea erhält währenddessen immer wieder durch L initiierte Unterstützungsangebote, am Spiel teilzunehmen. Im abschließenden Gesprächskreis wird das Spiel evaluiert und der Blick auf die nächste Stunde gerichtet.

\footnotetext{
4 Wir weisen den Förderbedarf mit Blick auf die Analyseeinstellung explizit nicht aus.
} 
Auf den ersten Blick nähert sich das Beispiel verschiedenen im Inklusionsdiskurs vertretenen Programmatiken an: Der Unterricht folgt einem inklusionspädagogisch befürworteten Prinzip gemeinsamen Unterrichts an gemeinsamen Gegenständen (z. B. Feuser 1982), in diesem Fall das gemeinsame Spielen des Spiels Brennball, also ,,angepasste gemeinsame Aktivitäten“ (Tiemann 2015, S. 56). Dabei steht das Spielen selbst im Vordergrund, nicht etwa der kritisch diskutierte Erwerb spezifischer Fertigkeiten oder Fähigkeiten (Giese und Weigelt 2013). Hierbei kommt es gerade nicht zum „,bloßen Nebeneinander“ (Frühauf 2008, S. 19) von Schüler*innen mit und ohne zugeschriebenem ,Förderbedarf“, sondern zu einem „Miteinander“ (ebd.), das - wie es einige Ansätze vorschlagen (Giese und Weigelt 2015a, S. 15) - stark an den Schüler*innen mit den vermeintlich größten Handicaps ausgerichtet ist.

Unser zweiter, videoanalytisch ,verlangsamter" und praxistheoretisch fokussierter Blick kann nun aufzeigen, dass und wie genau solche Programmatiken von um Inklusion bemühten Sportunterricht in spannungsvoller Relation zu „der sich prozessierenden Privilegierung und Marginalisierung bestimmter Schülergruppen“ (Wagener 2018, S. 98) stehen. Die Untersuchung weist darauf hin, dass bestimmte Ein-/Ausschlussmechanismen praktisch gerade deshalb so wirksam sind, weil sie auf einen wiederholt aktualisierten, weitgehend implizit geteilten Orientierungsrahmen gründen. Im Beispiel sind die Inklusionsbemühungen der Akteur*innen mit praktischen Differenzkonstruktionen und Ein-/Ausschlüssen verbunden, die einem dominanten, kollektiven Orientierungsrahmen folgen. Dieser wurde als Form der Personifikation unterrichtlicher Handlungen rekonstruiert und strukturiert den fallspezifischen interaktiven Erfahrungsraum: Ein zentrales - für den Inhaltsbereich „Spielen“ typisches - Handlungsproblem besteht im Beispiel darin, das Brennballspiel ans Laufen zu bringen und am Laufen zu halten, dass alle Schüler*innen mitspielen können. Die praktische Bearbeitung dieser Handlungsanforderung erfolgt in einer Form, in der die Handlungen von L sowie der ,Regelschüler*innen“ wiederholt und kollektiv daran orientiert sind, Lea - die Schülerin mit zugeschriebenem ,Förderbedarf' - gleichsam mit allen Mitteln ins Spiel zu integrieren. Dies führt zu einer Zentrierung des Unterrichts auf Lea sowie zu ihrer wiederholten Exponierung und Besonderung. Die Untersuchung kann detailliert aufzeigen, dass sich mit dieser Differenzordnung für die unterschiedlichen Akteur*innen je spezifische Handlungs- und Beteiligungsmöglichkeiten figurieren. Deutlich wird, wie diese Ein-/ Ausschlussprozesse in parallelen Interaktionen multimodal hervorgebracht werden, sich vielschichtig ausdifferenzieren und auch körperlich und materiell getragen werden, z. B. durch Artefakte oder Gesten, die Leas Besonderung permanent indizieren und stabilisieren. Zudem wird der Orientierungsrahmen kollektiv verwirklicht, was bedeutet, dass neben L auch die Schüler*innen substanziell zur Herstellung der Differenzordnung beitragen.

Diese Befunde werden nun an ausgewählten Sequenzen exemplarisch aufgezeigt. Ausgehend vom angesprochenen Handlungsproblem, möchten wir drei zentrale Dimensionen von Prozessen der Differenzherstellung und -bearbeitung im kollektiven Erfahrungsraum des formal inklusiven Grundschulsportunterrichts herausstellen und diskutieren: die Reifizierung von Un-/Fähigkeit (4.1), die Komplementarität von Handlungsräumen (4.2) und die Verschränkung von Differenz- und Sachkonstruktionen (4.3). 


\subsection{Reifizierung von ,Unfähigkeit`}

Die erste Dimension beschreibt, wie sich eine Differenzordnung dadurch bildet, dass ein ,formaler Förderbedarf' in der praktischen Bearbeitung unterrichtlicher Handlungsprobleme als leitende, auf ,Leistung' bezogene Differenzkategorie reifiziert wird. Für den ,Klassenraumunterricht' konnte verschiedentlich gezeigt werden, dass Schüler*innen mit ,sonderpädagogischem Förderbedarf' besonders von Benachteiligungen durch eine praktisch zugeschriebene bzw. hervorgebrachte , ungenügende Leistungsfähigkeit‘ oder ,Unfähigkeit‘ betroffen sind (vgl. Budde et al. 2016). Die folgenden Sequenzen illustrieren, dass solche Prozesse auch im Sportunterricht greifen, hier aber eine besondere Form annehmen können.

Der Unterricht beginnt im rituellen Sitzkreis, nach einem gemeinsamen Erinnern und Klären der Brennball-Spielregeln dreht sich das Unterrichtsgespräch um Optionen auf Sonderregeln für die zwei $S^{*}$ mit ausgewiesenem ,Förderbedarf ‘ und schließlich Leas diesbezügliche Entscheidung:

L: Eine besondere Regel gibt es noch diesmal. Weil ich gesehn hab dass manche Kinder schon auf den großn Kastenklettern und manche brauchen einen kleinen Kasten. Du darfst dir bei diesem Hindernis ((zeigt auf zwei nebeneinanderstehende Kästen unterschiedlicher Größe)) aussuchn. Schaff ichs auf den großn Kasten oder möchte ich den kleinen Kasten benutzn. Tobi, du darfs dir aussuchn. Möchtes du auf den Kastn klettern oder möchtes du mit einer Fliese laufen und immer wenn ich trommel musst du auf der Fliese stehn. Was möchtes du lieber?

Tobi: Kasten

L: Lea möchtest du auf den Kastn klettern auf die Matte ((zeigt)) oder möchtes du mit einer Fliese laufn und immer wenn ich trommel musst du draufstehn? ((hält Lea die Fliese hin)) (2) Das möchtes du? Möchtes du die oder die? ((zeigt)).

Lea: Da ((zeigt)).

L: Die? ((zeigt auf eine Fliese)).

Lea: Ja

L: Bitteschön. (4)

Die Lehrerin führt zunächst eine an alle adressierte Regel zur Binnendifferenzierung ein: Die $S^{*}$ können im Spielverlauf individuell entscheiden, ob sie bei einem Hindernis einen kleinen, , leichter ${ }^{`}$ zu erklimmenden Kasten den großen Kästen vorziehen. Es folgt eine zweite Modifikation, die L exklusiv Lea und Tobi als Wahlmöglichkeit unterbreitet: Die beiden müssen auf der Stelle zwischen der Nutzung der Hindernisse und einer Teppichfliese als tragbares ,Hindernis“ auswählen. Tobi wählt die für alle angebotene und in dieser Hinsicht ,normale' Spielposition (Kasten). Lea optiert dagegen für eine Fliese und wird so praktisch zur einzigen Person mit exklusiven Sonderregeln. Diese verändern Leas Handlungsaufträge im Spiel und werden durch das Angebot verschiedenartiger Fliesen weiter personalisiert. Vor dem Hintergrund der Ausrichtung des Unterrichts auf das gemeinsame Spielen des Spiels Brennball zeigt sich somit, wie die beschriebene Adressierung der Lehrkraft, die Annahme einer exklusiven Spielposition und die ,schweigende ' kollektive Ratifizierung 
des hiermit aufgerufenen Rahmens durch die übrige Klasse Lea als eine Lernende positionieren, die in Hinblick auf die sich scheinbar von selbst verstehenden grundlegenden Leistungsanforderungen des Unterrichtsgegenstandes - der Spielablauf des Brennballspiels, der Brennballparcours mit seinen Hindernishöhen und Laufdistanzen - ungenügend mitspielfähig und daher gesondert unterstützungsbedürftig ist. In dieser Form der Besonderung reifiziert sich ihr formal zugewiesener ,Förderbedarf ‘ auf der Ebene der praktisch wirksamen Differenzkategorie ,Leistung ' als eine kollektiv geltend gemachte ,ungenügende Fähigkeit' bzw. ,Unfähigkeit‘ in Relation zu aufgerufenen Normen und Normalitäten des sportunterrichtlichen Lernens.

Im Stundenverlauf werden Fliese und Trommel zu bleibenden materiellen Indikatoren und Stabilisatoren von Leas Besonderung. Vor allem nimmt diese Differenzsetzung eine ganz spezifische Form an, die - wie die nächste Sequenz veranschaulicht in wiederholten Praktiken der gesonderten Zuwendung hergestellt wird.

Nachdem L zwei Teams eingeteilt, die jeweilige Spielrolle (Wurfteam, Fangteam) zugewiesen und die Startpositionen markiert hat, steht kurze Zeit später das Wurfteam Team in einer Reihe hintereinander neben einer blauen Matte auf der einen Hallenseite. Das Fangteam steht verteilt in der Mitte der Turnhalle, wobei ein Schüler neben einem Kasten (dem Brennmal) kniet. L begibt sich zur Hallenseite und erinnert auf dem Weg dorthin Lea an ihre Sonderregel:

L: ((zu Lea gerichtet)) Lea? Wenn ich trommel ne. Wenn ich so mach ((Lw haut mehrfach auf die Trommel in ihrer Hand)) musst du ganz schnell auf deiner Fliese stehn. Wolln wir mal üben? Eins zwei drei huh schnell schnell schnell schnell schnell. Fliese runter und. (.) Sehr gut. Wenn ich nich mehr trommel darfs du laufen. ((zeigt mit der Hand in die Laufrichtung)). (2) Du läufst. Wolln wir das einmal abgehen?

Lea: Ja.

L: Komm. (.) Nimm deine Fliese. (.) Wir laufn. Hier hier hier hier hier hier hier hier hier hier hier. (2) und hierum. ((zeigt)) und weiter weiter weiter weiter ${ }^{\circ}$ weiter weiter ${ }^{\circ}$ ((flüsternd)) hierauf ((zeigt)) (2) wupp. Und hierum. ((schnellatmend)). (4) Und was is wenn ich trommel? ((trommelt)). huuh. Was passiert?

Los los los. ${ }^{\circ}$ Die Fliese ${ }^{\circ}$ ((flüsternd)).

Lea: Fliese.

L: Ja los. Mach runter. ((trommelt)). (2) Sehr gut. Und wir laufn weiter (1) Fliese nehm. (4) Und jetzt darfs du dich wieder hinten anstelln. ((zeigt)).

$\mathrm{S}^{*}:(($ Klatschen $))$

L: ((während die anderen $\mathrm{S}^{*}$ klatschen)) Jaa alles gut. Da müssn wir nich klatschn. (2) Wo has du gestandn? Such mal deinen Platz wieder?

Was als beiläufige Erinnerung beginnt, entwickelt sich zu einem Sonderdurchlauf, der Lea erneut ins Zentrum des Unterrichts setzt und durch die begleitenden Wertungen (,sehr gut“) öffentlich eine distinkte Leistungsfähigkeit und Leistung konstruiert. Parallel dazu entsteht eine Wartezeit für die übrige Klasse, die sie (zwar stimmlos aber sinngemäß) univok und weitgehend rahmenkonform füllt: Die $S^{*}$ stehen wartend am Platz, beschäftigen sich vereinzelt mit kurzen Tanzeinlagen aus dem Computerspiel „Fortnite“, versuchen durch Klatschen auf die Vorderbühnenaktivität von L und Lea Bezug zu nehmen und stellen dieses sofort wieder ein, als 
es von $\mathrm{L}$ als rahmenüberschreitend markiert wird (,da müssn wir nich klatschn“). Dieses konforme (Ab-)Warten validiert die Sonderzuwendung von $\mathrm{L}$ und damit die fortgesetzte Besonderung von Lea, die parallelen Interaktionen erweisen sich als komplementär. Zugleich positioniert es die übrige Klasse kurzfristig als ,Statisten “ am Rand der Bühne des Unterrichts. Propositional und performativ bleibt auf diese Weise Leas Unterschiedlichkeit und ,Un-Fähigkeit' zentral. Hier zeigt sich ein gängiges unterrichtliches Muster in der Strukturlogik von besser/schlechter (vgl. z.B. Wagener 2018, S. 88 f.), mit dem im ,Klassenraumunterricht' meist Marginalisierungen der vermeintlich schlechteren bzw. ,unfähigen' $S^{*}$ durch räumliche Absonderung, zeitliche Verzögerung oder Auslagerung auf Nebenschauplätze des Unterrichts einhergehen (z.B. Sturm und Wagner-Willi 2015). Dagegen nimmt im vorliegenden Fall die durch Unterstützungsmaßnahmen hergestellte leistungsbezogene Besonderung von Lea bemerkenswerterweise die Form einer Zentralisierung an: Sie rückt - im Stundenverlauf immer - in den Mittelpunkt des Geschehens, der Unterrichtsfortgang ist an ihr orientiert und es sind die bereits ,Spielfähigen', die warten müssen.

Dieser Befund regt zu einer differenzierteren Untersuchung und Bestimmung der Differenzkategorie ,Leistung ' bzw. ,Dis-/Ability' insbesondere auch mit Blick auf fachliche Spezifiken des Sportunterrichts an: In welcher Hinsicht verweisen die dargestellte Form der Reifizierung von ,Unfähigkeit', die Ausgestaltungen und Funktionen von Unterstützungsbemühungen sowie die Bedeutungen materieller Praxisbestandteile auf Charakteristika etwa des Inhaltsbereichs „Spielen“, des Unterrichtsprinzips ,gemeinsam an gemeinsamen Gegenständen“, des Grundschulsports und/oder des Sportunterrichts im Allgemeinen?

\subsection{Komplementäre Handlungsräume}

In der letztbehandelten Sequenz wurde bereits anschaulich, dass die übrige Klasse, auch und gerade dann, wenn sie praktisch in den Hintergrund tritt, maßgeblich an der performativen Hervorbringung von Leas ,Un-Fähigkeit“ mitwirkt. Die praxistheoretische Unterrichtsforschung betont, dass praktisch vollzogene In-/Exklusionen nie eindimensional zu verstehen sind und weder unidirektional verlaufen noch nur eine Person betreffen (z. B. Wagener 2018). Eine zweite Dimension handlungspraktischer Ein-/Ausschlussprozesse setzt hier an und verweist darauf, Differenzerzeugungen und -bearbeitungen stärker mit Blick auf die Relationalität der unterschiedlichen Handlungs- und Beteiligungsmöglichkeiten zu betrachten, die sich einzelnen Schüler*innen oder -gruppen eröffnen (vgl. hierzu auch Sturm et al. 2020). Im Beispiel erzeugen die Handlungen aller Akteur*innen, die durch den beschriebenen kollektiv getragenen Rahmen orientierten sind, differente aber komplementär aufeinander bezogene Möglichkeitsräume für Lea und ,die Klasse'. Die Relationalität von Ein-/ Ausschlussprozessen zeigt sich fallspezifisch als Komplementarität:

Das Brennballspiel ist in Gang gekommen und die Meisten wissen offenbar, was sie zu tun haben. L agiert auf einer Bank am Rand stehend als Spielleiterin, sie trommelt, gibt wiederkehrend Kommandos (,Stop“) oder erinnert Spielregeln und -aufgaben (,loslaufen“). Die meisten Kommentare bleiben auf Lea ausgerichtet („Lea, auf die Fliese“). Nach zwei Minuten unterbricht L das Spiel: 
L: Stopp stopp stopp stopp. Alle mal leise (2) ((in Richtung von Lea)) Lea, versuch auch mal. Wenn der Ball fliegt dann darfs du laufn okay? (.) Wenn ich trommel musst du ganz schnell wieder da stehn sonst bist du auch irgendwann raus okay? (.) Was- Wann darfs du laufn?

Lea: ((spricht unverständlich))

L: Nochmal? ((geht zu Lea hin)).

Lea: Fußball. Wenn der Fußball wirft.

L: Nein wenn Lars wirft. Und wenn der Ball fliegt dann darfs du laufn und wenn der Ball dort ist ((zeigt auf das Brennmal)) dann muss du stehenbleibn.

Versuch mal okay?

Lea: Ja

L: Los geht's.

Die kollektive Spielunterbrechung - alle kommen Ls Stopp-Anweisung unmittelbar nach - eröffnet erneut Zeit und (akustischen) Raum für eine exklusive Interaktion zwischen L und Lea. Wieder steht Lea im Scheinwerferlicht einer von L geführten Extrazuwendung, die um eine Integration Leas in die Spielabläufe bemüht ist. Dabei sind Ls Bemühungen darauf gerichtet, Lea sowohl mit Blick auf die Spielregeln (,versuch auch mal“) als auch auf die Konsequenzen bei Übertretung (,,bist du auch irgendwann raus“) in eine normale Spielposition zu führen. Der hier kommunikativ aufgerufenen Programmatik, dass für Lea im Grunde ,irgendwann“ scheinbar normale Regeln greifen sollen, steht - im Sinne des eingangs angesprochenen Spannungsverhältnis von Programmatik und Praxis - die öffentliche und performative Markierung und Etablierung ihrer Noch-Nicht-Fähigkeit als soziale Funktion von Ls Adressierungen gegenüber. Der so konturierte Handlungsraum wird praktisch durch die erneute Passivität des rahmenkonformen Warteverhaltens der übrigen $\mathrm{S}^{*}$ ermöglicht und gestützt, die gleichzeitig komplementäre Möglichkeitsräume eröffnet: Den $S^{*}$ tun sich einerseits - wie oben etwa am Beispiel aufgeführter Tänze aus dem beliebten Computerspiel „Fortnite“ bereits deutlich wurde - Spielräume für peerkulturelle Aktivitäten und Hinterbühnenhandlungen auf, während andererseits die Verwirklichung von Spielinteressen in dieser Situation praktisch ausgeschlossen ist.

Der weitere Spielverlauf ist wiederholt durch derartige, mitunter sehr kurz aufeinander folgende Unterbrechungen gekennzeichnet. Auch werden wenig später einzelne $S^{*}$ als „Helfer“ (L) für Lea abgestellt, die sie an die Hand nehmen, mit ihr den Parcours durchlaufen und sie an die einzelnen Handlungsschritte (laufen, stoppen, auf die Fliese stellen) erinnern sollen. Damit gehen spezifische Erwartungshaltungen und Möglichkeiten der Auseinandersetzung mit Mitschüler*innen sowie der Sache Brennball einher, während Leas Zentralisierung beibehalten und auf Dauer gestellt wird. Letzteres erfolgt auch darüber, dass sie von L ins andere Team transferiert wird, als Fang- und Wurfteam die Rollen tauschen. Insgesamt wird so das Ein-/Ausschlussmuster einer relationalen Verbindung von Besonderung und Behinderung verstetigt und ausdifferenziert: Das kollektive Bemühen einer Integration Leas in das Spiel birgt nicht nur eine exponierte Teilnahmeposition für sie, sondern ist handlungspraktisch mit einer komplementären spielbezogenen Behinderung der Übrigen verbunden. Weiterführend verweist diese Dimension darauf, dass noch kein 
systematisches Wissen darüber vorliegt, wie Ein-/Ausschlussrelationen handlungspraktisch unterrichtliche Aktivitäten auf den jeweiligen Bühnen ermöglichen oder verschließen. Schließlich ist Leas Zuwendung durch L exklusiv, die peer-Aktivitäten hingegen finden ohne sie statt.

\subsection{Verschränkung von Differenz- und Sachkonstruktionen}

Eine dritte wichtige Dimension sportunterrichtlicher Differenzkonstruktionen, die wir abschließend hervorheben möchten, ist in den bisherigen Ausführungen schon verschiedentlich aufgeschienen und bedarf keiner weiteren Beispielsequenz. Sie beschreibt die konstitutive Verschränkung von Differenz- und Sachkonstruktionen, von doing difference und doing content. In der praxistheoretischen Unterrichtsforschung werden Differenzkonstruktion verschiedentlich auch in Überlagerung und Abhängigkeit zur Hervorbringung anderer Ordnungskategorien untersucht, etwa Konstruktionen von Geschlechterverhältnissen oder Peer-Kultur (z. B. Sturm und WagnerWilli 2015). Nur vereinzelt werden Hervorbringungsprozesse und Konstruktionsweisen des Unterrichtsgegenstandes, der Sache, hier ganz explizit mitgeführt (etwa bei Reh und Rabenstein 2013). Unsere Analysen legen es nahe, sportunterrichtliche Differenzbildungen konsequent in Bezug zu Formen und Mustern der Sachhervorbringung und -ausdeutung zu betrachten. Im Fallbeispiel stellt sich, anders als in anderen Unterrichten (z. B. ebd.), das Zeigen der Sache durch die Lehrerin stärker als ein gemeinsames Erinnern und interaktives Aufführen eines bekannten Spiels dar. Die spezifische Hervorbringung und Ausdeutung der Sache Brennball ergibt sich hier in starker Bezogenheit auf die gezeigten Ein- und Ausschlussmechanismen: Im Vordergrund des Unterrichts steht nicht das Vorankommen (im Brennballspiel), sondern dessen Modifikation als für alle verbindliche Sache, um Lea grundlegend spielfähig, bzw. Elemente des Spiels ,lea-fähig' zu machen und ihr auf diese Weise eine Auseinandersetzung mit dem Fachinhalt zu ermöglichen. Dies bringt im Rahmen der thematisierten Wartezeiten für die Übrigen wiederholt eine temporäre Entkopplung aus den Spielrollen sowie ein kollektives In-den-Hintergrundtreten des in der Spielidee angelegten Wettspielcharakters mit sich. Nimmt man die idealtypische Spielidee als Vergleichshorizont, werden - deskriptiv und wertungsfrei - in diesem Unterricht keine Punkte für erfolgreich absolvierte Runden gezählt und aufaddiert. Genauso wenig stellte sich am Ende die Frage nach Siegern*innen und Verlierer*innen, einem fachkulturell stark ausgeprägten Merkmal von Sportunterricht (z.B. Zander 2018). Auch kommunikativ werden im Abschlusskreis der Beispielstunde inhaltliche Anpassungen des Spiels thematisiert: Die $S^{*}$ wünschen sich für das nächste Mal etwa „schwerere Hindernisse“ oder Brennball „mit Schießen“ und damit Adaptionen, die innerhalb des personifizierenden Rahmens offenbar praktikabel scheinen und so schließlich auch von L und der Klasse als gemeinsame - und damit Leakompatible - Perspektive festgehalten werden.

Damit verweist die Untersuchung zum einen darauf, die inklusionspädagogische Forderung nach gemeinsamem Unterricht an gemeinsamen Gegenständen insofern zu problematisieren, als dass wir für den Sportunterricht in der Grundschule noch viel zu wenig darüber wissen, wie sich welche (Lern-)Gegenstände praktisch konstituieren, welchen Modi dies folgt und welche Gehalte z. B. für ein Spiel geltend 
gemacht werden. Vor diesem Hintergrund kann zum anderen für eine praxistheoretische (Sport-)Unterrichtsforschung plädiert werden, die dem Verhältnis von Differenzerzeugung und Sachkonstruktion noch mehr Gewicht beimisst. Perspektivisch könnte es darum gehen, stärker in den Blick zu nehmen, auf welche Weise sich Unterricht in der Grundschule als Fachunterricht konstituiert. Angesprochen ist damit nicht nur die Frage nach sachbezogenen Differenzkonstruktionen, sondern auch nach differenzbezogenen Sachkonstruktionen im Grundschulsportunterricht, für die es (in Zukunft weiterer) empirisch-systematischer Vergleichshorizonte bedarf.

\section{Perspektiven praxistheoretischer inklusionsbezogener Sportunterrichtsforschung}

In einer praxistheoretischen Perspektiven wurde gezeigt, inwiefern mit unterrichtlichen Bemühungen der Inklusion ,förderbedürftiger' Schüler*innen im Grundschulsportunterricht wiederrum praktische Differenzkonstruktionen und Ein-/ Ausschlussprozesse einhergehen. Es ist davon auszugehen, dass solcherlei Prozesse unweigerlich Bestandteil von Unterrichtspraxis, den Beteiligten aber weitegehend unbewusst sind. Ihre systematische Untersuchung kann deshalb - fernab jeder wissenschaftlichen ,Besserwisserei ${ }^{\text {- }}$ - ein wichtiges Reflexionswissen generieren (Wagener 2018, S. 89), das für den formal inklusiven Grundschulsport und den Sportunterricht im Allgemeinen noch eine Leerstelle markiert. Am Fallbeispiel wurden die Reifizierung von ,Unfähigkeit', die Komplementarität von Handlungsräumen sowie die Verschränkung von Sach- und Differenzkonstruktionen als drei Dimensionen herausgestellt und bereits eingehender diskutiert, die auch über den Fall hinaus Fragen der sportunterrichtlichen Differenzherstellung spezifizieren können. Dabei konnte - dies scheint angesichts des stark individuumsorientierten sportpädagogischen Forschungsdiskurses besonders bedeutsam - aufgezeigt werden, dass die Differenzordnungen, die den spezifischen interaktiven Erfahrungsraum des Unterrichts kennzeichnen, auf erstens implizite, zweitens kollektive und drittens routinemäßig verwirklichte Orientierungen verweisen: Was sich im Beispiel programmatisch als ,,angepasste gemeinsame Aktivitäten“ (Tiemann 2015) fassen lässt, zeigt sich handlungspraktisch als Bearbeitung des Handlungsproblems, das Spiel Brennball dann so ans Laufen zu bringen und am Laufen zu halten, dass alle mitspielen (können). Im Horizont der rekonstruierten Figur der Zentrierung des Unterrichts auf Lea ist weder austauschbar noch irrelevant, dass hier ,ne Runde Brennball“ gespielt wird, ,alle mal leise“ sein sollen und es dann auch sind, wenn Lea rumgeführt wird. Das implizite - und im Fallbeispiel stark kollektivierte - Wissen darüber, wie Brennball gespielt wird, wie sich zu verhalten ist, wenn Lea eine Sonderzuwendung bekommt, usw. erweist sich handlungspraktisch als vorgeordnet und wirkt auf diese Weise an der Etablierung und Neukonstituierung der Differenzordnung - in Bezug auf die Sache des Unterrichts - mit. Der kollektiv geteilte Orientierungsrahmen der „Personifizierung unterrichtlicher Handlungen“ stiftet auf diese Weise unbewusst und interaktiv einen Erfahrungsraum, der konjunktives Wissen um Differenz und um die Sachen des Sportunterrichtes bereithält. Innerhalb dieses Rahmens wird die Unfähigkeit Leas kollektiv aktualisiert und differenziert, womit Veränderungen 
der Sache Brennball und spezifische Beteiligungs(un)möglichkeiten einhergehen, die sich als ambivalent für das Kollektiv zeigen. Das analytische Potenzial der vorgestellten Forschungsperspektive liegt darin, dieses kollektive Unbewusste der formal inklusiven Unterrichtspraxis zu explizieren und für ihre Vielschichtigkeiten, Widersprüchlichkeiten und Spannungen zu sensibilisieren.

Danksagung Wir bedanken uns herzlich bei Prof. Dr. Jan Erhorn, Leonie Möller und Wiebke Langer für die Bereitstellung der Daten.

Funding Open Access funding enabled and organized by Projekt DEAL.

Open Access Dieser Artikel wird unter der Creative Commons Namensnennung 4.0 International Lizenz veröffentlicht, welche die Nutzung, Vervielfältigung, Bearbeitung, Verbreitung und Wiedergabe in jeglichem Medium und Format erlaubt, sofern Sie den/die ursprünglichen Autor(en) und die Quelle ordnungsgemäß nennen, einen Link zur Creative Commons Lizenz beifügen und angeben, ob Änderungen vorgenommen wurden.

Die in diesem Artikel enthaltenen Bilder und sonstiges Drittmaterial unterliegen ebenfalls der genannten Creative Commons Lizenz, sofern sich aus der Abbildungslegende nichts anderes ergibt. Sofern das betreffende Material nicht unter der genannten Creative Commons Lizenz steht und die betreffende Handlung nicht nach gesetzlichen Vorschriften erlaubt ist, ist für die oben aufgeführten Weiterverwendungen des Materials die Einwilligung des jeweiligen Rechteinhabers einzuholen.

Weitere Details zur Lizenz entnehmen Sie bitte der Lizenzinformation auf http://creativecommons.org/ licenses/by/4.0/deed.de.

\section{Literatur}

Asbrand, B., \& Martens, M. (2018). Dokumentarische Unterrichtsforschung. Wiesbaden: Springer VS.

Block, M.E., Giese, M., \& Ruin, S. (2017). Inklusiver Sportunterricht - eine internationale Standortbestimmung. Sonderpädagogische Förderung heute, 62(3), 233-243.

Bohnsack, R. (2010). Rekonstruktive Sozialforschung. Einführung in qualitative Methoden. Opladen: Budrich.

Bohnsack, R. (2017). Praxeologische Wissenssoziologie. Opladen: Budrich.

Buchner, T., Pfahl, L., \& Traue, B. (2015). Zur Kritik der Fähigkeiten: Ableismus als neue Forschungsperspektive der Disability Studies und ihrer Partner_innen. Zeitschrift für Inklusion, 9 (2). https://www. inklusion-online.net/index.php/inklusion-online/article/view/273. Zugegriffen: 15. Sept. 2020.

Budde, J., \& Hummrich, M. (2013). Reflexive Inklusion. Zeitschrift für Inklusion, 7 (4). https://www. inklusion-online.net/index.php/inklusion-online/article/view/193. Zugegriffen: 15. Sept. 2020.

Budde, J., Blasse, N., \& Johannsen, S. (2016). Praxistheoretische Inklusionsforschung im Schulunterricht. Zeitschrift für Inklusion, 10 (4). https://www.inklusion-online.net/index.php/inklusion-online/article/ view/358. Zugegriffen: 15. Sept. 2020.

Erhorn, J., Langer, W., \& Möller, L. (2020). Vorbereitung angehender Sportlehrkräfte auf einen inklusiven Sportunterricht? Eine kritische Bestandsaufnahme hochschuldidaktischer Lehrformate. German Journal of Exercise and Sport Research, 50(3), 487-500. https://doi.org/10.1007/s12662-020-006685.

Feuser, G. (1982). Integration = die gemeinsame Tätigkeit (Spielen/Lernen/Arbeiten) am gemeinsamen Gegenstand/Produkt in Kooperation von behinderten und nichtbehinderten Menschen. Behindertenpädagogik, 21(2), 86-105.

Frühauf, T. (2008). Von der Integration zur Inklusion - ein Überblick. In A. Hinz, I. Körner \& U. Niehoff (Hrsg.), Von der Integration zur Inklusion. Grundlagen, Perspektiven, Praxis (S. 11-32). Marburg: Lebenshilfe-Verlag.

Giese, M. (2019). Konstruktionen des (Im-)Perfekten. Skizze einer inklusiven Fachdidaktik im Spiegel der Disability Studies (Schriften der Deutschen Vereinigung für Sportwissenschaft, Band 281). Hamburg: Czwalina. 
Giese, M., \& Ruin, S. (2018). Forgotten bodies - an examination of physical education from the perspective of ableism. Sport in Society, 21(1), 152-165. https://doi.org/10.1080/17430437.2016.1225857.

Giese, M., \& Weigelt, L. (2013). Sportunterricht auf dem Weg zur Inklusion. Sportpädagogik, 37(6), 2-5.

Giese, M., \& Weigelt, L. (2015a). Konstituierende Elemente einer inklusiven Sportdidaktik. In Dies (Hrsg.), Inklusiver Sportunterricht. Theorie, Empirie, Praxis (S. 10-52). Aachen: Meyer \& Meyer.

Giese, M., \& Weigelt, L. (Hrsg.). (2015b). Inklusiver Sportunterricht. Theorie, Empirie, Praxis. Aachen: Meyer \& Meyer.

Giese, M., \& Weigelt, L. (Hrsg.). (2017). Inklusiver Sport- und Bewegungsunterricht. Theorie und Praxis aus Sicht der Förderschwerpunkte. Aachen: Meyer \& Meyer.

Haegele, J. A., Giese, M., Wilson, W. J., \& Oldörp, F. (2020). Bruchlinien der Inklusion. German Journal of Exercise and Sport Research, 50(3), 417-425. https://doi.org/10.1007/s12662-020-00663-w.

Herzmann, P., \& Merl, T. (2019). Inklusion und dis/ability: Überlegungen zu einer Differenztheoretischen Unterrichtsforschung. Zeitschrift für Inklusion, 13 (2). https://www.inklusion-online.net/index.php/ inklusion-online/article/view/531. Zugegriffen: 15. Sept. 2020.

Lindmeier, C., \& Buchner, T. (2019). Zur Einführung: Grundzüge, Rezeptionslinien und Desiderate ableismuskritischer Forschung im deutschen Raum. Sonderpädagogische Förderung heute, 12(3), 233-239.

Mannheim, K. (1964). Beiträge zur Theorie der Weltanschauungsinterpretation. In K. Mannheim (Hrsg.), Wissenssoziologie (S. 91-154). Neuwied: Luchterhand. Original veröffentlicht 1922.

Merl, T. (2019). un/genügend fähig. Zur Herstellung von Differenz im Unterricht inklusiver Schulklassen. Bad Heilbrunn: Verlag Julius Klinkhardt.

Reh, S., \& Rabenstein, K. (2013). Die soziale Konstitution des Unterrichts in pädagogischen Praktiken und die Potentiale qualitativer Unterrichtsforschung. Rekonstruktionen des Zeigens und Adressierens. Zeitschrift für Pädagogik, 59(3), 291-307.

Ruin, S., \& Meier, S. (2018). Fragt doch mal uns! - Potenziale und Herausforderungen im inklusiven Sportunterricht aus Schülerperspektive. Leipziger Sportwissenschaftliche Beiträge, 59(1), 67-87.

Ruin, S., Meier, S., Leineweber, H., Klein, D., \& Buhren, C. G. (Hrsg.). (2016). Inklusion im Schulsport. Anregungen und Reflexionen. Weinheim: Beltz.

Schatzki, T.R. (2002). The site of the social. A philosophical account of the constitution of social life and change. Pennsylvania State University Press: University Park.

Sturm, T. (2016). Rekonstruktiv-praxeologische Schul- und Unterrichtsforschung im Kontext von Inklusion. Zeitschrift Für Inklusion, 10 (4). https://www.inklusion-online.net/index.php/inklusion-online/ article/view/321. Zugegriffen: 15. Sept. 2020.

Sturm, T., \& Wagner-Willi, M. (2015). Praktiken der Differenzbearbeitung im Fachunterricht einer integrativen Schule der Sekundarstufe. Zur Überlagerung von Schulleistung, Peerkultur und Geschlecht. Gender - Zeitschrift für Geschlecht, Kultur und Gesellschaft, 7(1), 64-78.

Sturm, T., Wagener, B., \& Wagner-Willi, M. (2020). Inklusion und Exklusion im Fachunterricht. Ambivalente Relationen in Schulformen der Sekundarstufe 1. In I. v. Ackeren, H. Bremer, F. Kessl, H. C. Koller, N. Pfaff, C. Rotter, D. Klein \& U. Salaschek (Hrsg.), Bewegungen. Beiträge zum 26. Kongress der DGfE (S. 581-595). Opladen: Budrich.

Tiemann, H. (2015). Inklusiven Sportunterricht gestalten - didaktisch-methodische Überlegungen. In M. Giese \& L. Weigelt (Hrsg.), Inklusiver Sportunterricht in Theorie und Praxis (S. 53-66). Aachen: Meyer \& Meyer.

Wagener, B. (2018). Inklusion aus Perspektive der praxeologischen Wissenssoziologie. In T. Sturm \& M. Wagner-Willi (Hrsg.), Handbuch Schulische Inklusion (S. 77-92). Opladen: Budrich.

Wagner-Willi, M., \& Sturm, T. (2012). Inklusion und Milieus in schulischen Organisationen. Zeitschrift für Inklusion, 6 (4). https://www.inklusion-online.net/index.php/inklusion-online/article/view/32. Zugegriffen: 15. Sept. 2020.

Zander, B. (2018). Sportunterricht als konjunktiver Erfahrungsraum. Rekonstruktion kollektiver Orientierungen zum Sportunterricht von Schüler_innen im 7. Schuljahr. Zeitschrift für sportpädagogische Forschung, 6(2), 5-30. 